Bull. Austral. Math. Soc.

Vol. 41 (1990) [337-342]

\title{
ENTIRE FUNCTIONS THAT SHARE FINITE VALUES WITH THEIR DERIVATIVES
}

\author{
YANG LIAN-ZHONG
}

It is shown that if a nonconstant entire function $f(z)$ and its derivative $f^{(k)}(z)$, share two finite values (counting multiplicities), then $f(z)=f^{(k)}(z)$, where $k$ is a positive integer.

\section{INTRODUCTION}

We say that two nonconstant entire functions $F(z)$ and $G(z)$ share the value $a$ provided that $F=a$ if and only if $G=a$; we distinguish among shared values $\mathrm{CM}$ (counting multiplicities) and shared values IM (ignoring multiplicities). A famous theorem of Nevanlinna [4, Theorem 2.6] implies that if two nonconstant entire functions $f$ and $g$ on the complex plane share four distinct values IM then it follows that $f=g$. When we consider the special case $g=f^{\prime}$, the derivative of $f$, Rubel and Yang proved the following result $[6]$.

THEOREM A. If a nonconstant entire function $f$ and its derivative $f^{\prime}$ share two finite values $C M$, then $f=f^{\prime}$.

This suggests the following conjecture:

(a) The "CM" can be replaced by "IM" in Theorem A.

(b) The "entire" can be replaced by the word "meromorphic" in Theorem A.

(c) The function $f^{\prime}$ can be replaced by $f^{(k)}$ in Theorem A.

Recently, (a) has been proved by Mues and Steinmetz [5] and (b) has been proved by Gundersen $([2,3])$. This paper concerns conjecture $(c)$ and proves

Theorem 1. Let $f(z)$ be a nonconstant entire function. If 0 is a Picard value of both $f$ and $f^{(k)}$, and $f$ shares a finite value $a \neq 0 I M$ with $f^{(k)}(k \geqslant 2)$, then $f=e^{A z+B}$, where $A$ and $B$ are constants with $A^{k}=1$.

THEOREM 2. If a nonconstant entire function $f(z)$ and $f^{(k)}(z)$ share two finite values $C M$, then $f=f^{(k)}$.

Received 6 April 1989

This research was partially supported by a grant from NSF.

Copyright Clearance Centre, Inc. Serial-fee code: 0004-9729/90 \$A2.00+0.00. 


\section{LEMMAS}

It is assumed that the reader is familiar with Nevanlinna theory and its notation $T(r, f), m(r, f), N(r, f), S(r, f)$, et cetera, as found in [4].

LEMMA 1. [6]. Let $f(z)$ be a nonconstant meromorphic function in $|z|<\infty$. If 0 is a Picard value of both $f$ and $f^{(k)}(k \geqslant 2)$. Then $f=e^{A z+B}$ or $f=(a z+b)^{-n}$, where $a, b, A, B$ are constants with $a A \neq 0$.

Lemma 2. $[4$, p.62]. Suppose that $a$ and $b \neq 0$ are finite complex numbers, and $f(z)$ is a nonconstant meromorphic function in $|z|<\infty$. Then

$$
T(r, f) \leqslant\left(2+\frac{1}{k}\right) N\left(r, \frac{1}{f-a}\right)+\left(2+\frac{2}{k}\right) N\left(r, \frac{1}{f^{(k)}-b}\right)+S(r, f) .
$$

Lemma 3. [4]. Let $f(z)$ be a nonconstant meromorphic function in the complex plane; then

$$
\begin{aligned}
m\left(r, \frac{f^{(k)}}{f}\right) & =S(r, f) \\
T\left(r, f^{(k)}\right) & \leqslant(k+1) T(r, f)+S(r, f)
\end{aligned}
$$

\section{Proofs OF THEOREMS}

Proof of Theorem 1: Since 0 is a Picard value of both $f$ and $f^{(k)}$, by Lemma 1 we have $f(z)=e^{A x+B}$, where $A \neq 0$ and $B$ are constants.

On the other hand, $f$ shares $a \neq 0$ IM with $f^{(k)}$, by Lemma $2, a$ can not be a Picard value of both $f$ and $f^{(k)}$, therefore we can choose a complex number $z_{0}$ such that

$$
a=f\left(z_{0}\right)=e^{A z_{0}+B}
$$

so that

$$
a=f^{(k)}\left(z_{0}\right)=A^{k} e^{A z_{0}+B} .
$$

This implies $A^{k}=1$. Theorem 1 is thus proved.

COROLLARY. Suppose that $f$ satisfies all conditions of Theorem 1; then $f=f^{(k)}$.

Proof of Theorem 2: Suppose that $f$ shares two finite values $a$ and $b$ CM with $f^{(k)}$; we consider the following two cases.

CASE (1). $a b \neq 0$. Set $g=(f-a) /\left(f^{(k)}-a\right)$, then

$$
\frac{g^{\prime}}{g}=\frac{f^{\prime}}{f-a}-\frac{f^{(k+1)}}{f^{(k)}-a}=F_{1} \quad(\text { say })
$$


Since $f$ is entire and $a$ is a shared value CM by $f$ and $f^{(k)}$, therefore, the function $g$ has no zeros and poles. Hence we have

$$
\begin{aligned}
N\left(r, F_{1}\right) & \leqslant N\left(r, g^{\prime}\right)+N\left(r, \frac{1}{g}\right)=0 \\
m\left(r, F_{1}\right) & \leqslant m\left(r, \frac{f^{\prime}}{f-a}\right)+m\left(r, \frac{f^{(k+1)}}{f^{(k)}-a}\right) \\
& =S(r, f)+S\left(r, f^{(k)}\right)=S(r, f) \quad(\text { Lemma 3) }
\end{aligned}
$$

Thus it follows that

$$
T\left(r, F_{1}\right)=S(r, f)
$$

Similarly, if

$$
F_{2}=\frac{f^{\prime}}{f-b}-\frac{f^{(k+1)}}{f^{(k)}-b},
$$

then by the same reasoning

$$
T\left(r, F_{2}\right)=S(r, f)
$$

Suppose first that $F_{1} \not \equiv 0$ and $F_{2} \not \equiv 0$. Then from Nevanlinna's fundamental estimate and Jensen's Theorem we obtain

$$
\begin{aligned}
\frac{F_{1}}{f-a} & =\frac{f^{\prime}}{(f-a)(f-b)}-\frac{f^{(k+1)}}{f^{(k)}\left(f^{(k)}-b\right)} \cdot \frac{f^{(k)}}{f-a} \\
& =\frac{1}{b-a}\left(\frac{f^{\prime}}{f-b}-\frac{f^{\prime}}{f-a}\right)-\frac{f^{(k)}}{f-a} \cdot \frac{1}{b}\left(\frac{f^{(k+1)}}{f^{(k)}-b}-\frac{f^{(k+1)}}{f^{(k)}}\right) . \\
m\left(r, \frac{1}{f-a}\right) & \leqslant m\left(r, \frac{1}{F_{1}}\right)+S(r, f)+S\left(r, f^{(k)}\right) \\
& \leqslant T\left(r, F_{1}\right)+S(r, f)=S(r, f), \quad \text { (Lemma 3). }
\end{aligned}
$$

Similarly, by using $\left(F_{2}\right) /(f-b)$ we obtain

$$
m\left(r, \frac{1}{f-b}\right)=S(r, f)
$$

Now, if $f \not \equiv f^{(k)}$, then from the first fundamental theorem and the fundamental 
estimate we can get that

$$
\begin{aligned}
2 T(r, f) & =T\left(r, \frac{1}{f-a}\right)+T\left(r, \frac{1}{f-b}\right)+O(1) \\
& =N\left(r, \frac{1}{f-a}\right)+N\left(r, \frac{1}{f-b}\right)+S(r, f) \\
& \leqslant N\left(r, \frac{1}{\frac{f^{(k)}}{f}-1}\right)+S(r, f) \\
& \leqslant T\left(r, \frac{f^{(k)}}{f}\right)+S(r, f) \leqslant N\left(r, \frac{1}{f}\right)+S(r, f) \\
& \leqslant T(r, f)+S(r, f)
\end{aligned}
$$

which implies the contradiction $T(r, f)=S(r, f)$. Thus $f \equiv f^{(k)}$.

On the other hand, if $F_{1} \equiv 0$, then

$$
g=\frac{f-a}{f^{(k)}-a}=c
$$

where $c$ is some nonzero constant. Since $b \neq 0$ is not a Picard value for both $f$ and $f^{(k)}$ (Lemma 2), we can choose a complex number $z_{0}$ such that $f\left(z_{0}\right)=b=f^{(k)}\left(z_{0}\right)$. Therefore $c=1$, we obtain $f \equiv f^{(k)}$.

Similarly, if $F_{2} \equiv 0$, then $f \equiv f^{(k)}$. This proves $f \equiv f^{(k)}$ in case (1).

CASE (2). $a b=0$. We assume that $a=0$ and $b \neq 0$ without loss of generality. Set $h(z)=f /\left(f^{(k)}\right)$, then

$$
\frac{h^{\prime}}{h}=\frac{f^{\prime}}{f}-\frac{f^{(k+1)}}{f^{(k)}}=F_{3} \quad \text { (say) }
$$

Since $f$ is entire and shares $a=0 \mathrm{CM}$ with $f^{(k)}$, it follows that $h(z)$ has no poles and zeros, so we obtain

$$
\begin{aligned}
T(r, h) & =T\left(r, \frac{f}{f^{(k)}}\right)=T\left(r, \frac{f^{(k)}}{f}\right)+O(1)=S(r, f) \\
T\left(r, F_{3}\right) & =m\left(r, F_{3}\right)+N\left(r, F_{3}\right)=S(r, f)+N\left(r, \frac{h^{\prime}}{h}\right)=S(r, f) .
\end{aligned}
$$

Let $F_{2}$ be defined as in Case 1 ; then $T\left(r, F_{2}\right)=S(r, f)$.

We now proceed with the proof of Theorem 2 in the same way as in Case 1. Suppose 
first that $F_{2} \not \equiv 0$ and $F_{3} \not \equiv 0$. Since

$$
\begin{aligned}
\frac{1}{f-b} & =\frac{1}{F_{3}}\left(\frac{f^{\prime}}{f(f-b)}-\frac{f^{(k+1)}}{f(f-b)} \cdot \frac{f}{f^{(k)}}\right), \\
\frac{1}{f} & =\frac{1}{F_{2}}\left(\frac{f^{\prime}}{f(f-b)}-\frac{f^{(k+1)}}{\left(f^{(k)}-b\right) f^{(k)}} \cdot \frac{f^{(k)}}{f}\right), \\
T\left(r, \frac{f}{f^{(k)}}\right) & =T\left(r, \frac{f^{(k)}}{f}\right)+O(1)=S(r, f),
\end{aligned}
$$

we get

$$
m\left(r, \frac{1}{f-b}\right)=S(r, f), \quad m\left(r, \frac{1}{f}\right)=S(r, f)
$$

Now, if $f \not \equiv f^{(k)}$, then, from the first fundamental theorem, we have

$$
\begin{aligned}
2 T(r, f) & =N\left(r, \frac{1}{f}\right)+N\left(r, \frac{1}{f-b}\right)+S(r, f) \\
& \leqslant N\left(r, \frac{1}{f_{/ f}^{(k)}-1}\right)+S(r, f)+N\left(r, \frac{1}{f}\right) \\
& \leqslant T\left(r, \frac{f}{f^{(k)}}\right)+T(r, f)+S(r, f)=T(r, f)+S(r, f)
\end{aligned}
$$

which yield a contradiction. Thus $f \equiv f^{(k)}$.

On the other hand, if $F_{2} \equiv 0$, then by integrating $F_{2}$ we get

$$
\frac{f-b}{f^{(k)}-b}=c
$$

where $c$ is some nonzero constant. If $c=1$, then $f \equiv f^{(k)}$; if $c \neq 1$, then $a=0$ is a Picard value for both $f$ and $f^{(k)}$. This is impossible by the corollary of Theorem 1 .

Similarly, if $F_{3}=0$, then there is a nonzero constant $C$ such that $f \equiv C f^{(k)}$. If $C=1$, then $f \equiv f^{(k)}$. If $C \neq 1$, then $b \neq 0$ is a Picard value for both $f$ and $f^{(k)}$. This contradicts Lemma 2. This proves Theorem 2 in Case 2.

REMARK. The function $f=\sin z$ shares $0 \mathrm{CM}$ with $f^{\prime \prime}$, and $f \not \equiv f^{\prime \prime}$. It follows that the number two of Theorem 2 cannot be reduced. We do not know whether there are corresponding results to our theorems if multiplicities are ignored, or if meromorphic instead of entire functions are considered, but we know that our theorems are true if a meromorphic function with $N(r, f)=S(r, f)$ is considered. 


\section{REFERENCES}

[1] G. Frank, 'Eine vermutung von Hayman uber nullstellen meromorpher funktionen', Math. Z. 149 (1976), 29-36.

[2] G. Gundersen, 'Meromorphic functions that share finite values with their derivatives', $J$. Math. Anal. Appl. 75 (1980), 441-446.

[3] G. Gundersen, 'Meromorphic functions that share two finite values with their derivatives', Pacific J. Math. 105 (1983), 299-309.

[4] W.K. Hayman, Meromorphic Functions (Clarendon Press, Oxford, 1964).

[5] E. Mues and N. Steinmetz, 'Meromorphe funktionen, die mit ihrer ableitung werte teilen', Manuscripta Math. 29 (1979), 195-206.

[6] L.A. Rubel and C.C. Yang, 'Values shared by an entire function and its derivative', in Complex Analysis: Lecture Notes in Mathematics No. 599, (Proc. Conf.), pp. 101-103 (Springer-Verlag, Heidelberg, Berlin, New York, 1977).

Department of Mathematics

Shandong University

Jinan, Shandong 250100

People's Republic of China 